\title{
Measuring Molecular Diffusion Through Thin Polymer Films with Dual-Band Plasmonic Antennas
}

Hao Chen, ${ }^{\dagger}$ Gaurav Singhal, ${ }^{\dagger}$ Frank Neubrech, ${ }^{\ddagger}$, Runyu Liu,, Joshua S. Katz,, Scott Matteucci, ${ }^{\nabla}$ Steven G. Arturo, ${ }^{\circ}$ Daniel Wasserman,, Harald Giessen, ${ }^{\ddagger} \&$ Paul V. Braun,,$+\dagger$

${ }^{\dagger}$ Department of Material Science and Engineering, Materials Research Laboratory, and Beckman Institute for Advanced Science and Technology, University of Illinois Urbana-Champaign, Urbana, Illinois 61801, USA

¥4th Physics Institute and Research Center SCoPE, University of Stuttgart, Pfaffenwaldring 57, 70569 Stuttgart, Germany

${ }^{\S}$ Kirchhoff-Institute for Physics, University of Heidelberg, Im Neuenheimer Feld 227, 69120 Heidelberg, Germany

"Department of Electrical and Computer Engineering, University of Illinois Urbana-Champaign, Urbana, Illinois 61801, USA

"Formulation Science, Corporate Research and Development, The Dow Chemical Company, Collegeville, Pennsylvania 19426, United States

${ }^{\nabla}$ Formulation Science, Corporate Research and Development, The Dow Chemical Company, Midland, Michigan 48674, United States

${ }^{\circ}$ Engineering and Process Sciences, Corporate Research and Development, The Dow Chemical Company, Collegeville, Pennsylvania 19426, United States 
a

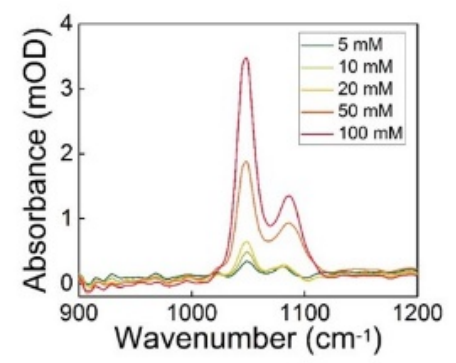

d

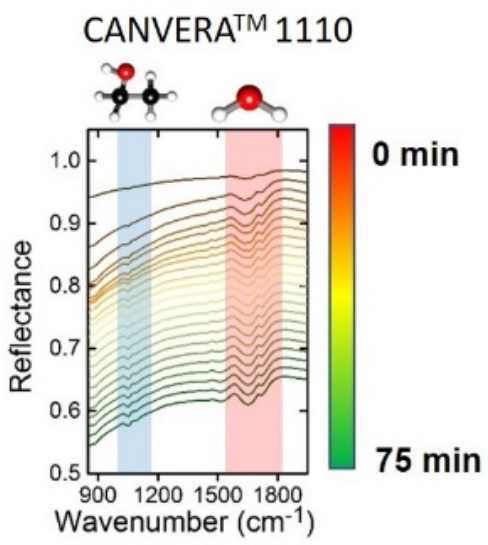

b

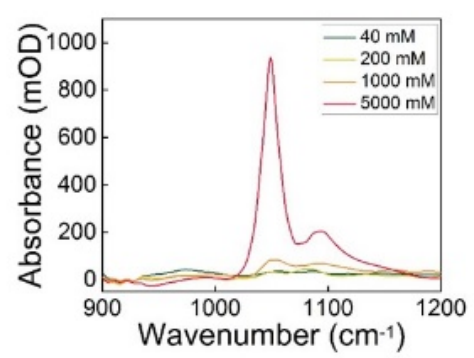

e

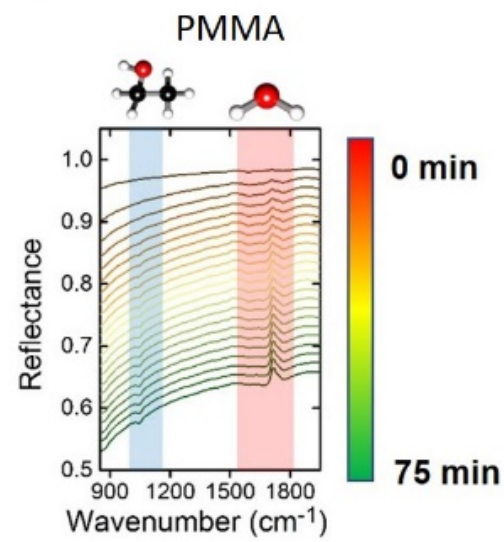

C

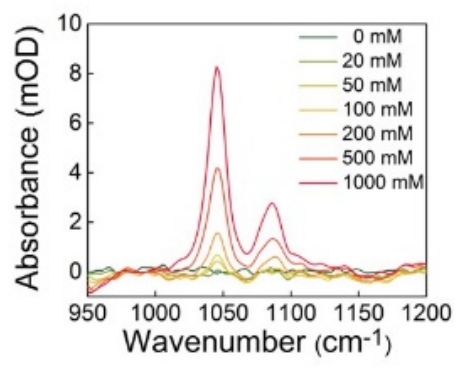

f

EXP-4599

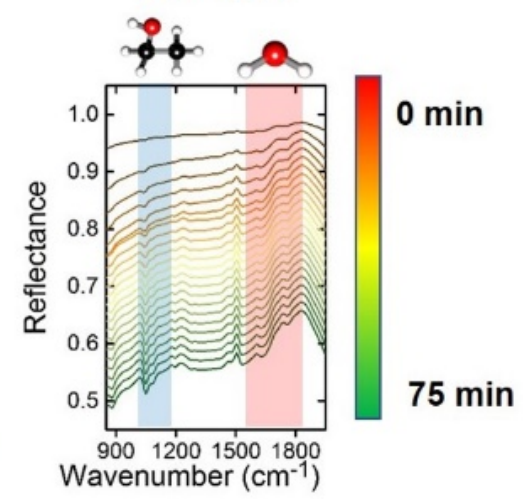

Figure S1. Absorbance spectra of ethanol as a function of concentration for (a) 0 to $100 \mathrm{mM}$ ethanol on a SEIRA substrate, (b) 40 to $5000 \mathrm{mM}$ ethanol on a bare substrate. (c) 20 to $1000 \mathrm{mM}$ ethanol with ATR system (d-f) Differential reflectance spectra as a $50 \mathrm{wt} \%$ ethanol water solution diffuses into 5- $\mu$ m-thick (d) CANVERA ${ }^{\mathrm{TM}} 1110$, (e) PMMA, and (f) EXP-4599 coatings. 

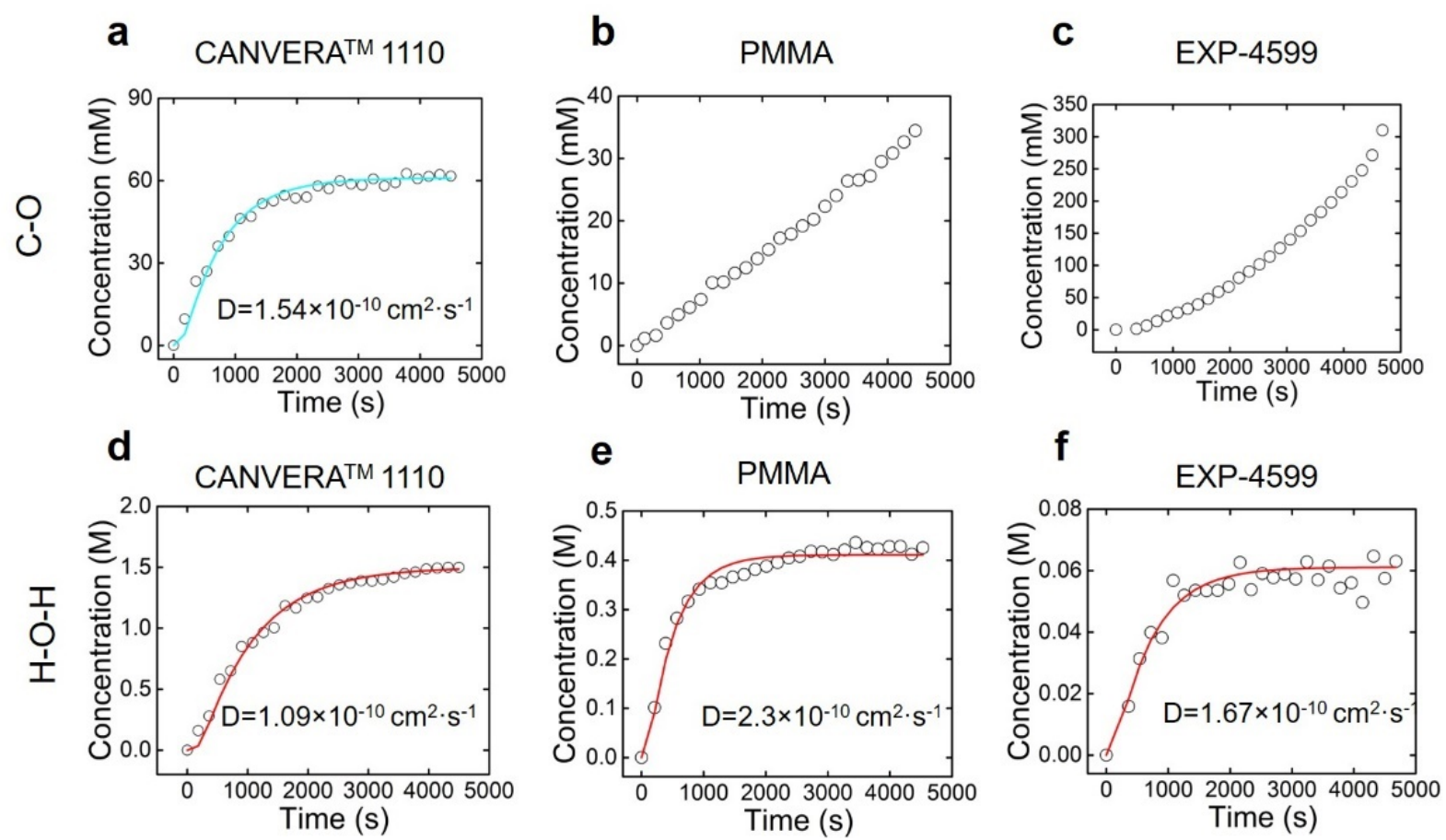

Figure S2. SEIRA-based measurement of ethanol and water diffusion into polymer coatings. Ethanol (a-c) and water (d-f) concentration as a $50 \mathrm{wt} \%$ ethanol water solution diffuses into CANVERA $^{\mathrm{TM}}$ 1110, PMMA, and EXP-4599. 
a

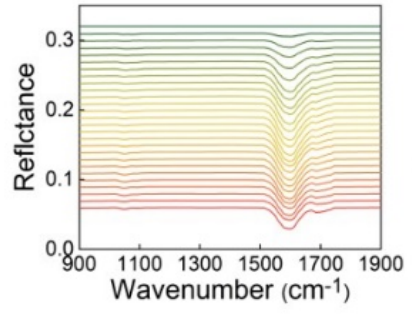

d

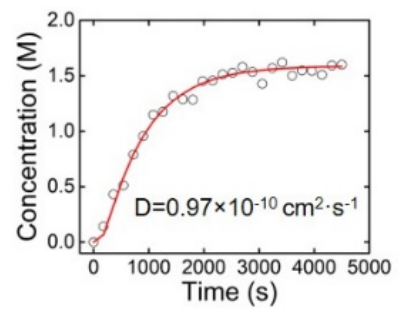

g

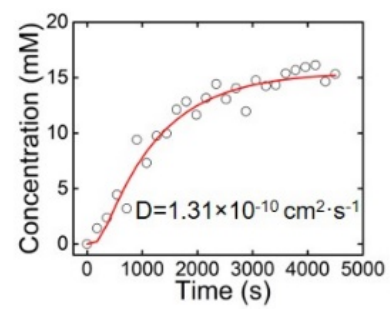

b

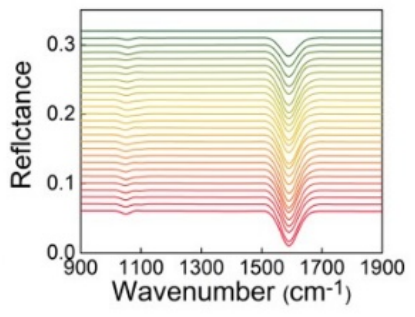

e

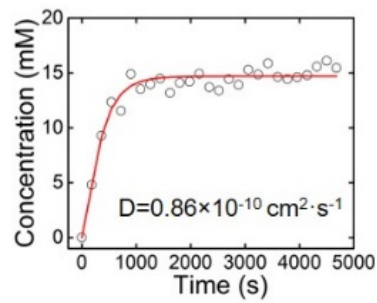

h

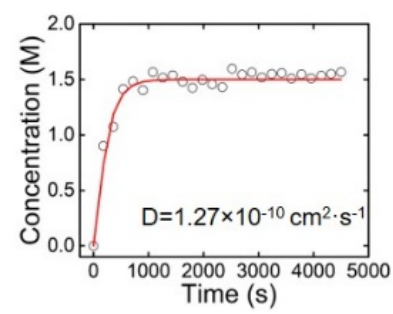

C

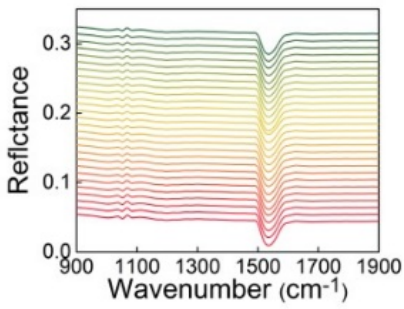

f

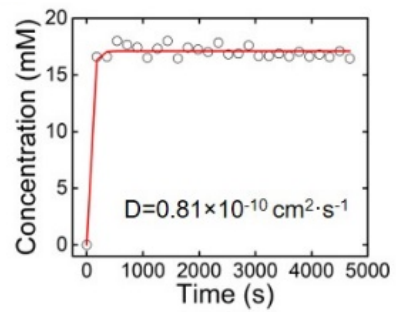

i

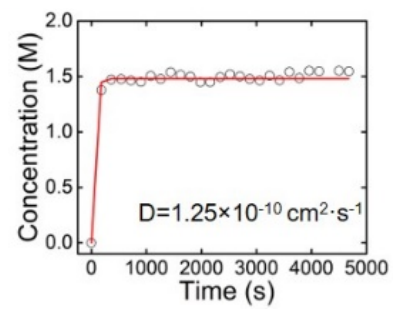

Figure S3. Differential reflectance spectra of $1 \mathrm{M}$ ethanol water solution into (a) 5- $\mu$ m-thick, (b) 2.5- $\mu$ m-thick (c) $0.5-\mu$ m-thick CANVERA ${ }^{\mathrm{TM}} 1110$ coatings. Concentration of ethanol in the film vs. time for (d) $5-\mu \mathrm{m}$-thick, (e) $2.5-\mu \mathrm{m}$-thick (f) $0.5-\mu$ m-thick CANVERA ${ }^{\mathrm{TM}} 1110$ coatings. Concentration of water in the film $v$ s. time for (g) $5-\mu \mathrm{m}$-thick, (h) $2.5-\mu \mathrm{m}$-thick (i) $0.5-\mu \mathrm{m}$-thick CANVERA $^{\mathrm{TM}} 1110$ coatings.
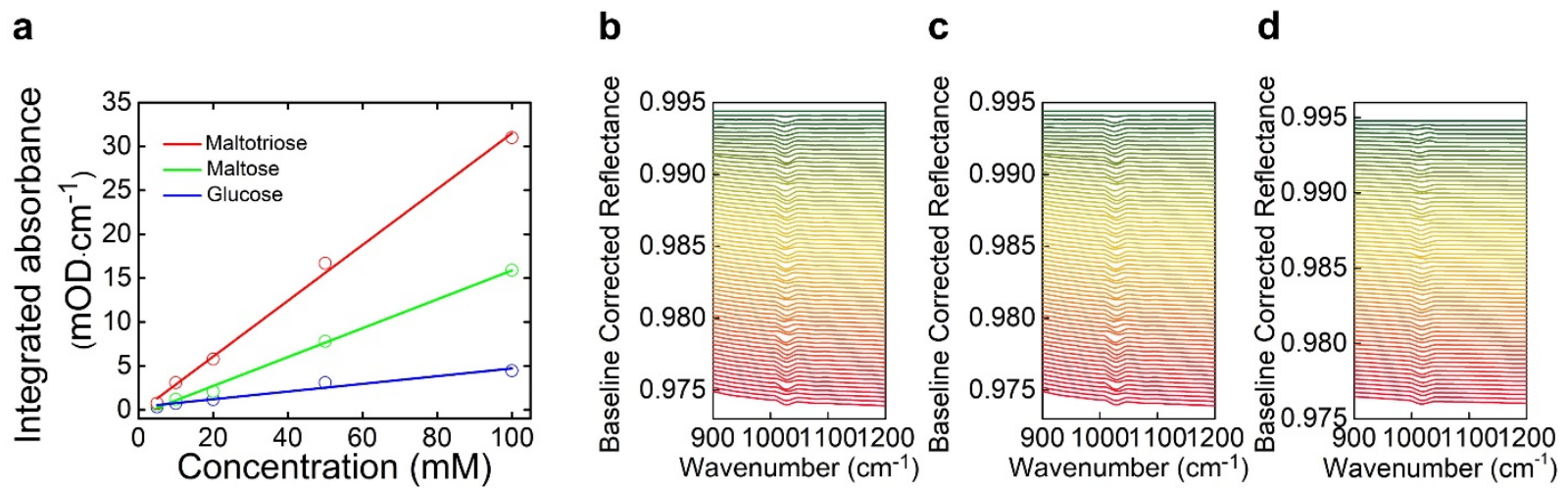

Figure S4. (a) Integrated absorbance calibration plot for 0 to $100 \mathrm{mM}$ glucose, maltose and maltrotriose. Differential reflectance spectra of $200 \mathrm{mM}$ glucose (b), maltose (c) and maltrotriose (d) water solution into $250 \mathrm{~nm}$-thick CANVERA ${ }^{\mathrm{TM}} 1110$ coating. 
a
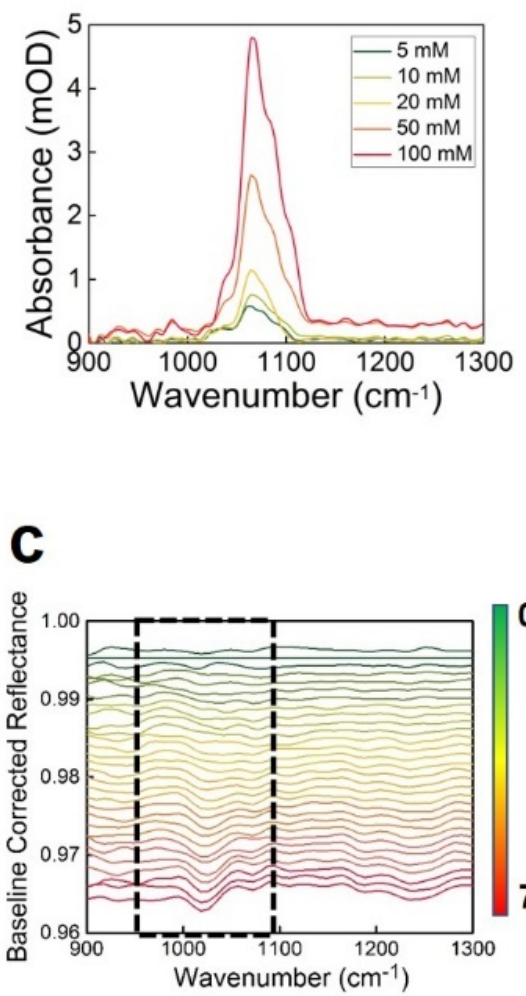

e

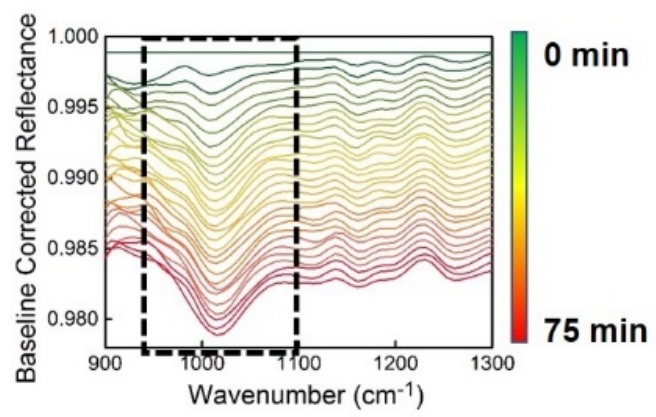

$0 \mathrm{~min}$ b

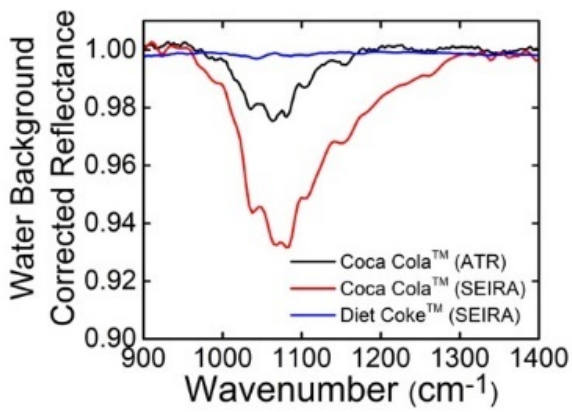

d

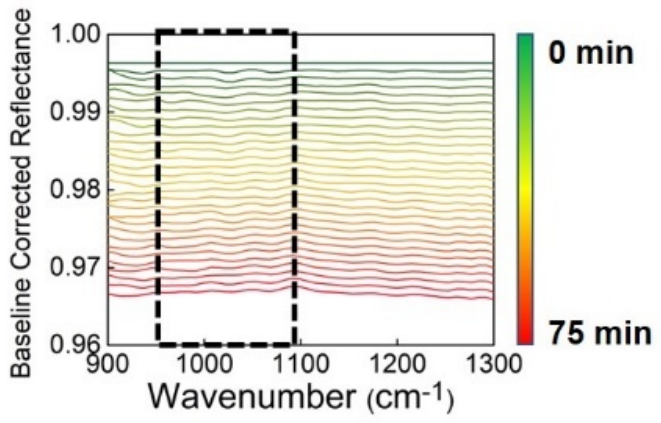

f

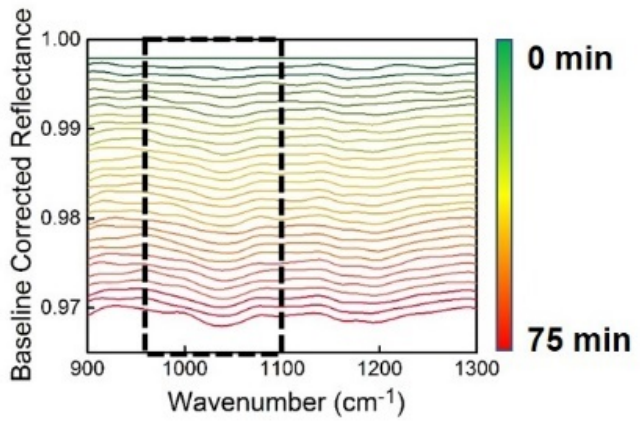

Figure S5. Coca Cola ${ }^{\mathrm{TM}}$ diffusion into CANVERA ${ }^{\mathrm{TM}}$ 1110, PMMA, and EXP-4599 coatings. (a) Absorbance spectra of fructose at concentrations from $0 \mathrm{mM}$ to $100 \mathrm{mM}$. (b) Reflectance of Coca-Cola $^{\mathrm{TM}}$ (red line) and Diet Coke ${ }^{\mathrm{TM}}$ (blue line) measured with SEIRA and Coca-Cola ${ }^{\mathrm{TM}}$ (black line) measured with ATR. (c-d) Differential reflectance spectra of Coca-Cola ${ }^{\mathrm{TM}}$ and Diet Coke $^{\mathrm{TM}}$ diffusing into 5- $\mu$ m-thick CANVERA ${ }^{\mathrm{TM}} 1110$ (e-f) Differential reflectance spectra of Coca-Cola ${ }^{\mathrm{TM}}$ diffusing into 5- $\mu$ m-thick PMMA (e) and EXP-4599 (f) coatings. 
a

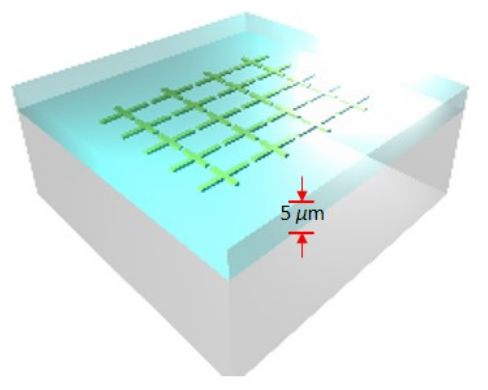

b

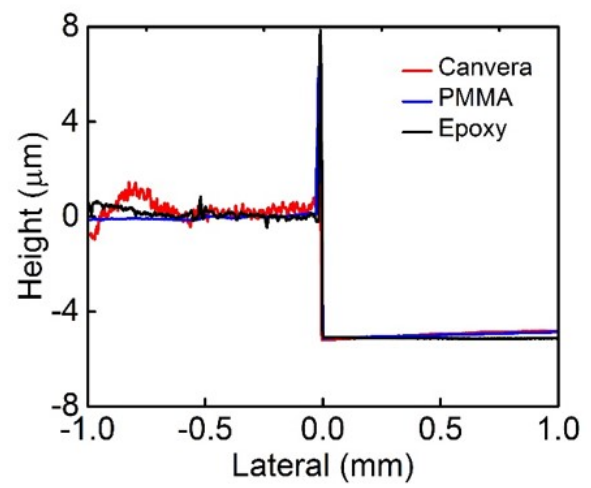

Figure S6. (a) Schematic of cross-nanoantenna sample coated with a 5- $\mu$ m thick polymer film. (b) 2D height profile of polymer film measured via profilometry (P-6 Stylus Profiler, KLATencor) across a scratch, giving the film thickness. The sharp peak right at the edge of the scratch is scratch residue.

a
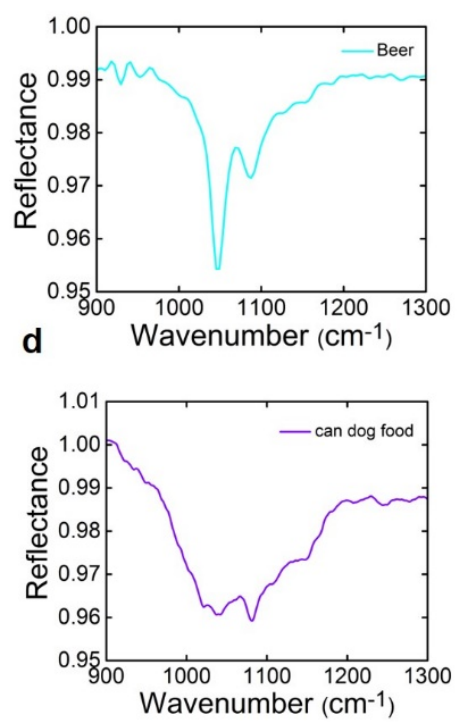

b
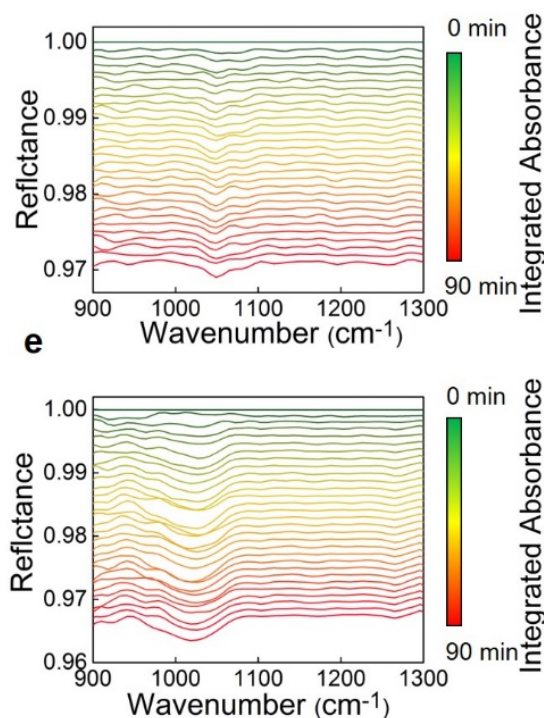

C
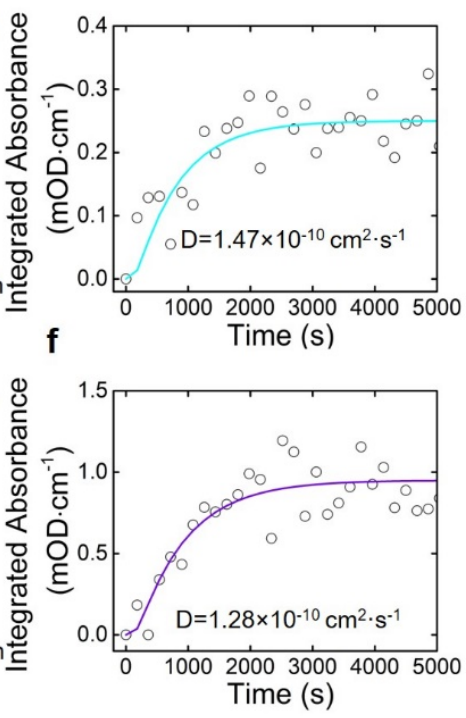

Figure S7. Beer (Bud Light ${ }^{\mathrm{TM}}$ ) and canned dog food (Alpo Prime Cut ${ }^{\mathrm{TM}}$ ) diffusion into $5 \mu \mathrm{m}$ thick CANVERA ${ }^{\mathrm{TM}} 1110$ films. (a) SEIRA reflectance spectra from beer. (b) Differential reflectance spectra of beer diffusing into the film over time (c) Integrated absorbance (units of $\mathrm{mOD} \cdot \mathrm{cm}^{-1}$ ) vs. time alcohol and carbohydrates. (d) SEIRA reflectance spectra plots of canned dog food. (e) Differential reflectance spectra of dog food diffusing into the film over time. (f) Integrated absorbance (units of $\mathrm{mOD} \cdot \mathrm{cm}^{-1}$ ) vs. time for dog food. In c, f experimental data are fit to a Fickian model. In a-c we assume we are measuring alcohol and carbohydrates compound (the peaks match what we expect for C-O vibration mode) and in d-f, we do not know what components of the dog food are diffusing into the film, thus plot integrated absorbance vs. time in $\mathrm{c}$ and $\mathrm{f}$. 


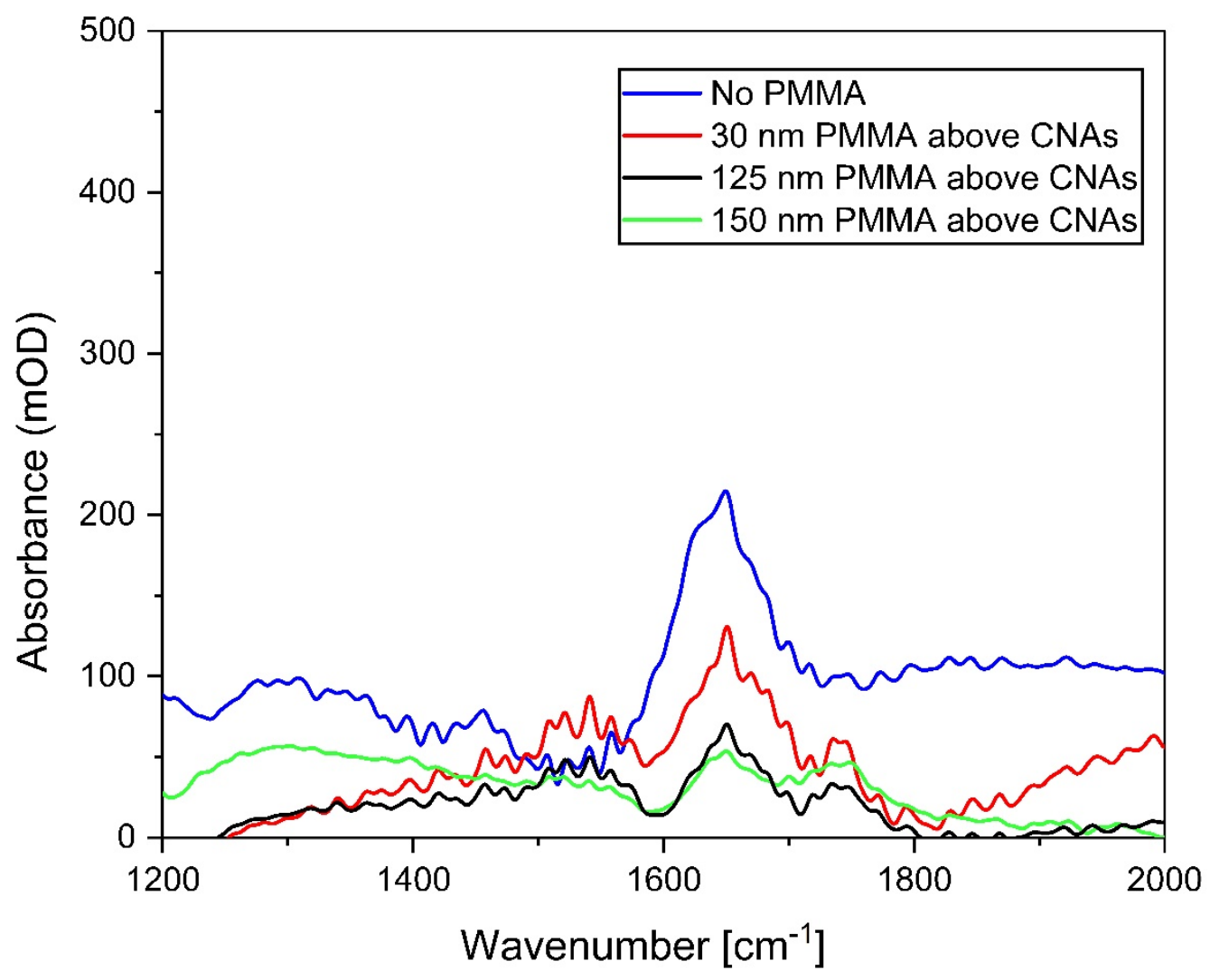

Figure S8. SEIRA-based measurement of water H-O-H scissoring absorbance after water was placed on bare CNAs and PMMA coated CNAs for PMMA thicknesses above the CNAs of 30 nm, $125 \mathrm{~nm}$ and $150 \mathrm{~nm}$ (see Figure S9 for how PMMA layer thickness above the antennas was determined). The background spectra subtracted from these spectra consisted of that collected from $\mathrm{D}_{2} \mathrm{O}$ above the same CNAs. $\mathrm{D}_{2} \mathrm{O}$ is used since it is not IR active at $\sim 1650 \mathrm{~cm}^{-1}$, but will swell the PMMA film to a similar degree as $\mathrm{H}_{2} \mathrm{O}$. This is important since PMMA swelling would reduce the concentration and thus absorbance of the PMMA in the near field of the CNAs. 
a

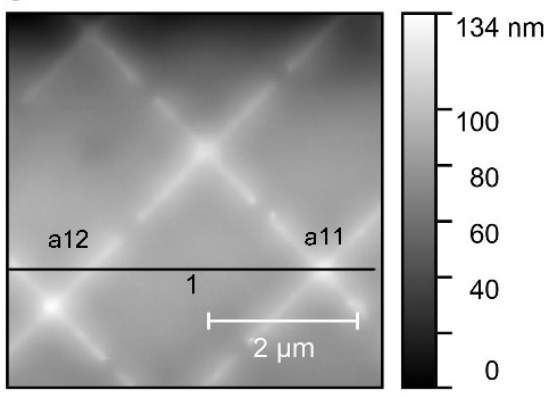

C

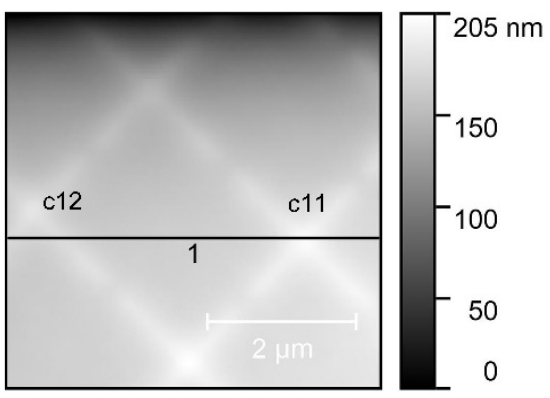

b

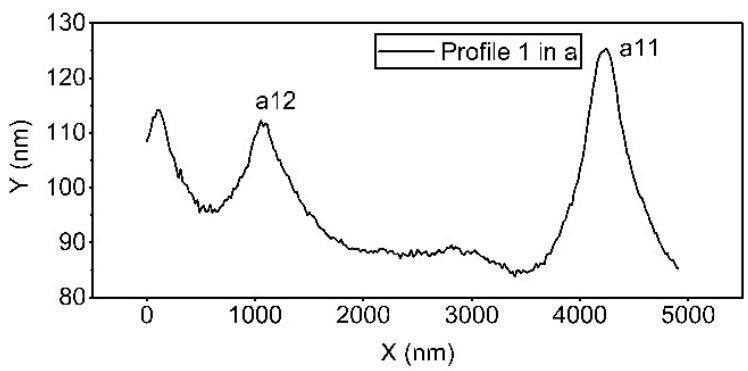

d

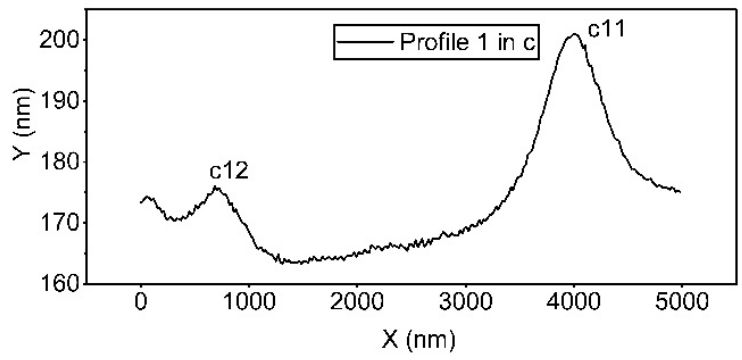

Figure S9. (a) AFM image of CNAs coated with a nominally $90 \mathrm{~nm}$ thick spin coated PMMA film. (b) Depth profile along line 1 in (a) with peaks at position a12 and a11 in (a). Peak at position a12 used to determine the thickness of the PMMA coating above the CNA (CNA is 80 $\mathrm{nm}$ high, the polymer is nominally $90 \mathrm{~nm}$ high, and the peak at a12 is $20 \mathrm{~nm}$ above the flat region of the PMMA, making the PMMA coating of the CNA $30 \mathrm{~nm}$. (c) AFM image of CNAs coated with a nominally $195 \mathrm{~nm}$ spin coated PMMA film. (d) Depth profile along line 1 in (c) with peaks at position $\mathrm{c} 12$ and $\mathrm{c} 11$ in (c). Peak at position c12 used to determine the thickness of the PMMA coating above the CNA (CNA is $80 \mathrm{~nm}$ high, the polymer is nominally $195 \mathrm{~nm}$ high, and the peak at a12 is $10 \mathrm{~nm}$ above the flat region of the PMMA, making the PMMA coating of the CNA 125 nm. AFM was not collected on the nominally $220 \mathrm{~nm}$ thick PMMA film, but given the trends observed, the PMMA layer above the CNA in that case should be 150 nm thick. Nominal PMMA thicknesses were measured via ellipsometry. 


\section{Supplementary Note 1:}

In this section, we studied what we presume is alcohol and perhaps carbohydrates from beer (Bud Light $^{\mathrm{TM}}$ ) diffusing into a CANVERA ${ }^{\mathrm{TM}} 1110$ coating. Beer is stored in open air for one hour to allow CO2 to diffuse out of the liquid. We observe a pronounced peak at $1040 \mathrm{~cm}^{-1}-1120$ $\mathrm{cm}^{-1}$ (Figure S7a) which can be assigned to vibrational modes associated with C-O bonds found both in ethanol and carbohydrates (Bud Light ${ }^{\mathrm{TM}}$ contains $4.2 \%$ alcohol and 6.6 grams of carbohydrates per $12 \mathrm{oz}$ ). While alcohol (ethanol) is lower in molecular weight than all carbohydrates, and thus probably diffuses faster into the coating, we cannot rule out that some of the signal is also due to carbohydrates. Differential absorbance spectra show the alcohol and carbohydrates diffuse into the CANVERA ${ }^{\mathrm{TM}} 1110$ coating (Figure S7b) and the combined alcohol and carbohydrate diffusion coefficient is calculated as $1.47 \times 10^{-10} \mathrm{~cm}^{2} \cdot \mathrm{s}^{-1}$ (Figure S7c).

Similar measurements are carried out for dog food (Alpo Prime $\mathrm{Cut}^{\mathrm{TM}}$ ) diffusing into CANVERA ${ }^{\mathrm{TM}}$ 1110. A broad peak appears at a $900 \mathrm{~cm}^{-1}-1150 \mathrm{~cm}^{-1}$, which can be assigned to vibrational modes associated with a combination of $\mathrm{C}-\mathrm{O}$ and $\mathrm{C}-\mathrm{O}-\mathrm{C}$ bonds. While we did not attempt to identify the composition of the molecules causing this peak, we suspect the absorption originates from a mixture of fats and proteins. Differential absorbance spectra demonstrate components of the dog food diffuse into the coating (Figure S7e) and the diffusion coefficient for these components can be fit via a Fickian model (Figure S7f) with $\mathrm{D}=1.28 \times 10^{-10} \mathrm{~cm}^{2} \cdot \mathrm{s}^{-1}$. Dog food is centrifuged to remove any solids and the remaining liquid is applied to the polymer coated chip.

\section{Supplementary Note 2:}

It is assumed that diffusion of active through material in this system is governed by 1D Fickian diffusion. ${ }^{\text {S1 }}$ The partial differential equation (PDE), the boundary conditions (BC), and the initial condition (IC) are given as the following

PDE: $\frac{\partial u}{\partial t}=D \frac{\partial^{2} u}{\partial z^{2}}$

BCs: $\left.\frac{\partial u}{\partial z}\right|_{z=0, t}=0$ and $u(z=L, t)=\bar{C}$,

IC: $u(z, t=0)=0$,

where $u(z, t)$ is the concentration of active at depth $z$ and time $t$. The BCs are chosen to keep a constant concentration of active at the interface with the solution and to have zero flux at the boundary at the substrate. The IC states that there is no active in the material at the beginning of the experiment.

Solutions methods and notation outlined in Farlow ${ }^{\mathrm{S} 2}$ are used to solve the PDE. The methods include applying transformations to make variables dimensionless and homogenizing the BCs. The latter allows for the use of separation of variables for an analytical solution. 
The analytical solution to the PDE in Equation 1 is

$u(z, t)=\bar{C}+\sum_{n=0}^{\infty} A_{n} e^{-\frac{-\lambda_{n}^{2} D}{L^{2}} t} \cos \frac{\lambda_{n} z}{L}$

where $\lambda_{n}=\frac{\pi}{2}+n \pi$ and $A_{n}=-\frac{2 \bar{C}}{\lambda_{n}} \sin \lambda_{n}$.

The concentration of the active in the region near the CNA, in the region between $z$ and $z_{d}$, is found by taking the integral of the analytical solution over this special range. This integral gives the following result

$S(t)=\int_{0}^{z} u(z, t) d z=\bar{C}+\frac{1}{z_{d}} \sum_{n=0}^{\infty} \frac{A_{n} L}{\lambda_{n}} e^{-\frac{-\lambda_{n}^{2} D}{L^{2}} t} \sin \left(\frac{\lambda_{n} z_{d}}{L}\right)$

where $\lambda_{n}$ and $A_{n}$ are given in Equation 2.

\section{Supplementary References}

S1. R. B. Bird, W. E. Stewart and E. N. Lightfoot, Transport Phenomena. Wiley: New York, 1960.

S2. S. J. Farlow, Partial Differential Equations for Scientists and Engineers. Dover: New York, 1993. 